\title{
La epidemiología de los factores de riesgo de los Síndromes coronarios agudos
}

\author{
Acute Coronary Syndrome: Epidemiology of risk factors
}

Las enfermedades crónicas no comunicables (ECNC) han desplazado a las enfermedades infecciosas como primera causa de morbimortalidad en nuestro país $(1,2)$; dentro de las ECNC, la enfermedad aterosclerótica vascular sistémica tiene una gran prevalencia y, los síndromes coronarios agudos (SCA) representan la expresión más dramática de la enfermedad isquémica coronaria. Los SCA se presentan generalmente en pacientes a partir de la quinta década de vida y están asociados a diferentes factores de riesgo que varían según la población estudiada. Por ejemplo, en sociedades occidentales (Norteamérica y Europa) se identifican como factores de riesgo mayores a la hipertensión arterial, la diabetes mellitus (DM), la hipercolesterolemia, el tabaquismo (activo y remoto), la edad y la historia familiar de enfermedad isquémica coronaria en edades tempranas. Existen también los factores de riesgo "secundario", pero no por ello menos importantes como: el estrés físico y emocional, el sedentarismo, la obesidad, el síndrome metabólico, la enfermedad apnea-sueño, y otros (3). Incluso, existen aplicativos digitales (cardioapp/ web) para el cálculo del riesgo de mortalidad por enfermedad cardiovascular.

En este número de la Revista, Hurtado y col. (4), nos presentan los resultados de un estudio observacional transversal de los SCA en 2 hospitales de la ciudad de Chiclayo con respecto a la incidencia y características clínicas de los mismos, así como un análisis de los factores de riesgo encontrados en esta población. Este estudio de 100 pacientes muestra sorprendentemente que el factor de riesgo más prevalente es la obesidad medida con el índice cintura-cadera, pero no con el IMC, seguida por la edad avanzada, la hipertensión arterial, la dislipidemia y el sedentarismo, todos ellos en más del $50 \%$ de la muestra, y el tabaquismo, la DM y la historia familiar de enfermedad coronaria prematura en un tercio o menos. Es decir, que los factores de riesgo "secundarios" adquieren un protagonismo importante en la ocurrencia de los SCA, ya que están directamente asociados a conductas y hábitos inadecuados en la población que, idealmente, podrían ser más fáciles de controlar con campañas educativas y motivacionales específicas, en contraposición a enfermedades crónicas ya establecidas que requieren de no solo cambios de estilo de vida sino también de intervención farmacológica para controlarlos, como la hipertensión y la diabetes.

Es también importante el análisis realizado con los hallazgos de este estudio en comparación con otros estudios realizados en el Perú y, específicamente Lima. La incidencia de sobrepeso y enfermedad metabólica es mayor en el norte del Perú que en la capital, sugiriendo diferencias no solo socio-económicas sino también ambientales; sería interesante la comparación con poblaciones en otras áreas geográficas, como las expuestas a las grandes alturas, y en la Amazonia.

En cuanto a prevención secundaria, es preocupante ver que un tercio de la población ya tuvo un evento coronario o cerebrovascular en el pasado y que no estaba recibiendo terapia anti plaquetaria o anti lipídica, que son fundamentales en pacientes vasculares declarados para evitar que las placas ateroscleróticas se vuelvan inestables y originen un nuevo cuadro agudo.

Como se mencionó anteriormente, este estudio es muy importante ya que identifica factores de riesgo que pueden ser relativamente fácil de controlar con intervenciones (5) o campañas educativas y motivacionales a nivel poblacional, sobre todo en niños (6), con un costo económico muy inferior al costo de tratamiento de la enfermedad una vez instalada y, en el caso específico de los SCA, un costo mucho mayor ya que requiere de infraestructura y terapias más sofisticadas y costosas, como la terapia fibrinolítica o, idealmente, la revascularización percutánea de emergencia, que muchas veces no son accesibles a la población general. 
La labor del médico, tanto general como especialista, es de educar a sus pacientes y a la población en general, en cómo prevenir la ocurrencia de enfermedades que pueden ser fatales.

\section{Alejandro García Revilla ${ }^{1, a, b}$}

\section{REFERENCIAS BIBLIOGRÁFICAS}

1. OPS Perú. Enfermedades no transmisibles y factores de riesgo. Lima: OPS Perú; 2018. [Internet]. (Citado el 09 de noviembre de 2019). Disponible en: https:// www.paho.org/per/index.php?option=com_co ntent\&view $=$ article $\&$ id $=4110$ :enfermedades-notransmisibles-y-factores-de-riesgo\&Itemid $=1062$

2. Enfermedades no transmisibles y transmisibles, 2017. Lima: INEI; 2017. [Internet]. (Citado el 09 de noviembre de 2019). Disponible en: https://www. inei.gob.pe/media/MenuRecursivo/publicaciones digitales/Est/Lib1526/index.html

3. Goff DC Jr, Lloyd-Jones DM, Bennett G, et al. 2013 ACC/AHA Guideline on the Assessment of Cardiovascular Risk. A Report of the American College of Cardiology/American Heart Association
Task Force on Practice Guidelines. Circulation. 2014; 129: S49-S73.

4. Hurtado E, Bartra A, Osada J, León F, Ochoa M. Frecuencia de factores de riesgo cardiovascular en pacientes con síndrome isquémico coronario agudo, Chiclayo. Rev Med Hered. 2019; 30:224-231.

5. European Society of Cardiology. Communitybased salt substitution programme lowers blood pressure. Sophia Antipolis, France: European Society of Cardiology; 2019. (Citado el 09 de noviembre de 2019). Disponible en: https:// www.escardio.org/The-ESC/Press-Office/ Press-releases/community-based-salt-substitutionprogramme-lowers-blood-pressure

6. Fernandez-Jimenez R, Al-Kazaz M, Jaslow R, Carvajal I, Fuster V. Children Present a Window of Opportunity for Promoting Health. J Am Coll Cardiol. 2018;72:3310-9.

1. Laboratorios de Hemodinámica e Intervesionismo Coronario. Hospital Regional St John's Oxnard, California. USA.

a. MD, FACC, FCAI. Especialista en Enfermedades Cardiovasculares.

b. Director 